\title{
La metodología ApS refuerza la adquisición de competencias generales y específicas
}

Teresa Perez Gil ${ }^{a}$, Nadia Martínez Gisbert ${ }^{b}$, Vera Soler Molinab ${ }^{b}$, Sara Puchades Díaz, Esther Giménez Martínez, Cristina Hurtado Vizcaíno y Dolores Ortiz-Masià

${ }^{a}$ Egresada del Grado de Logopedia de la Universitat de Valencia, ${ }^{b}$ Estudiante del Grado de Logopedia

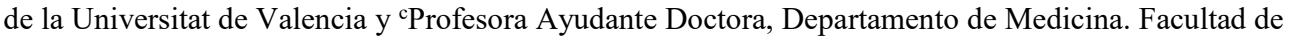
Medicina y Odontología. Universitat de Valencia (m.dolores.ortiz@uv.es).

\begin{abstract}
Service-learning (SL), through a process of action-reflection-action collaborates in an efficient and practical way to the consolidation of basic and specific competencies that contribute to a successful life and good social functioning. The aim of the work is to analyse how the SL methodology improves general and specific competences in the students. To this end, two experimental groups were formed: a) SL group; b) NO-SL group (control). Each of the groups was evaluated in specific competences (exploration of cranial pairs) and general competences (communication, adaptation,..). The results were compared using the $t$-Student test. The results showed that the SL group significantly improved, in a majority way, the competences evaluated in relation with the control group.
\end{abstract}

Keywords: speech therapy, service-learning, senior citizens, clinical propaedeutic, evaluation.

\section{Resumen}

El Aprendizaje-Servicio (ApS), mediante un proceso de acción-reflexiónacción colabora de manera eficiente y práctica a la consolidación de competencias básicas y específicas que contribuyen a una vida exitosa y al buen funcionamiento social. El objetivo del trabajo es analizar cómo la metodología ApS mejora competencias generales y especificas en el alumnado. Para ello se han formaron dos grupos experimentales: a) grupo $A p S$; b) grupo NO-ApS (control). Cada uno de los grupos fue evaluado en competencias específicas (exploración de pares craneales) y generales (comunicación, adaptación, etc.). Los resultados se compararon mediante el test $t$-Student. Los resultados mostraron que el grupo ApS mejoraban significativamente, de forma mayoritaria, las competencias evaluadas respecto al grupo control.

Palabras clave: aprendizaje-servicio, competencias especificas, competencias generales.

\section{Introducción}

El Aprendizaje-Servicio (ApS) representa el sumatorio resultante del aprendizaje y el servicio solidario, que al unirse generan una realidad nueva que intensifica los efectos de cada uno de ellos tomados por separado. El aprendizaje mejora el servicio ya que lo que se aprende se puede transferir en forma de acción y permite realizar un servicio de calidad a la comunidad. En el ApS se unen intencionalidad pedagógica e intencionalidad solidaria conformando un proyecto educativo de utilidad social. Además el ApS promueve valores, estimula la búsqueda y una adecuada jerarquización de valores como la prosocialidad, la 
mirada al mundo y la solidaridad con sus necesidades fundamentales (Rodríguez Gallego, 2014).

Por otro lado, el ApS, mediante un proceso de acción-reflexión-acción colabora de manera eficiente y práctica a la consolidación de competencias básicas y específicas que contribuyen a una vida exitosa y al buen funcionamiento social (Martínez, 2010).

Dentro de los programas de ApS se pueden distinguir cuatro tipos de servicios en los que concretan el aprendizaje: servicio directo, servicio indirecto, investigación y abogacía (Casado de la Gala, 2015).

La universidad dentro del Espacio Europeo de Educación Superior se impuso la obligación de orientar la educación y por tanto los resultados finales del aprendizaje durante un periodo formativo hacia la obtención de competencias susceptibles de ser evaluadas (European Higher Education Area and Bologna process)

El concepto de competencia hace referencia a "aptitud, pericia, idoneidad para hacer algo o intervenir en un asunto importante" pudiéndose utilizar como definición el "conjunto de conocimientos, habilidades, actitudes, comportamientos y actuaciones de un profesional", es decir, las competencias profesionales son capacidades construidas con componentes cognitivos combinados con habilidades psicomotoras y actitudes basadas en valores y creencias, que se expresan en conductas coherentes y previsibles. Así, las competencias se transforman en el elemento básico sobre el que analizar el perfil profesional de cada titulación y proponer un posible proyecto formativo o Plan de Estudios. Con las competencias se integran los tres pilares fundamentales que la educación superior debe desarrollar en los futuros titulados superiores: conocimientos, habilidades y actitudes.

Se pueden distinguir 4 tipos de competencias: básicas, transversales, generales y específicas.

- Las competencias básicas y las transversales no son propias de unos estudios concretos porque hacen referencia a aspectos necesarios para ejercer de forma adecuada cualquier profesión ya que son comunes a la mayor parte de profesiones y titulaciones universitarias. En el primer caso, están relacionadas con las capacidades intelectuales indispensables para el aprendizaje de una determinada profesión y se deben haber comenzado a adquirir en niveles educativos previos. Estas competencias pueden ser, a su vez, de varios tipos: instrumentales (capacidades cognitivas, metodológicas, tecnológicas y lingüísticas), interpersonales (capacidades individuales tales como habilidades sociales) y sistémicas (capacidades y habilidades relacionadas con sistemas globales como la combinación de comprensión, sensibilidad y conocimientos).

- Las competencias generales y específicas son propias de un ámbito o titulación y la distinguen de otras, por lo que están orientadas a la consecución de un perfil específico del Graduado. Estas competencias son motivo de especial atención durante el periodo de formación y su evaluación lleva al otorgamiento del título académico que reconoce socialmente su adquisición y la posibilidad del ejercicio de la profesión.

La formación de Grado en Logopedia está regulada por la ORDEN CIN/726/2009, de 18 de marzo, donde se establecen los objetivos y competencias que los egresados deben tener para el correcto desarrollo de la profesión. Dentro de los objetivos que cita dicha ORDEN se encuentra el objetivo específico "Explorar, evaluar, diagnosticar y emitir pronóstico de evolución de los trastornos de la comunicación y el lenguaje desde una perspectiva multidisciplinar, fundada en la capacidad de interpretación de la historia clínica para lo que se aplicarán los principios basados en la mejor información posible y en condiciones de seguridad clínica". El citado objetivo se implementa de forma concreta durante el desarrollo 
de la asignatura "Neurología Clínica Aplicada a la Logopedia". Para alcanzar el objetivo se deben adquirir competencias muy relacionadas con la evaluación y diagnóstico pero también deben adquirir competencias básicas como comunicar bien, empatía, trato correcto, entre otras.

En el presente proyecto se evalúan competencias relacionadas directamente con este objetivo específico de la asignatura, concretamente con la competencia específica 33 (CE33) que se encuentra en la memoria Verifica del Grado de Logopedia de la UV: "Evaluar, diagnosticar, pronosticar, rehabilitar y prevenir los trastornos de la comunicación asociados a disfunciones cognitivas".

De forma paralela el proyecto evalúa competencias generales del Grado de Logopedia, concretamente con las competencias generales que se encuentran en la memoria Verifica del Grado de Logopedia que se citan a continuación:

- CG7: Asesorar a las familias y al entorno social de los usuarios, favoreciendo su participación y colaboración en el tratamiento logopédico, atendiendo las peculiaridades de cada caso e incluyendo la perspectiva de género.

- CG13: Ser capaz de desarrollar habilidades como: regular su propio aprendizaje, resolver problemas, razonar críticamente y adaptarse a situaciones nuevas.

- CG18: Comunicar de manera oral y escrita sus observaciones y conclusiones al paciente, a sus familiares y al resto de profesionales que intervienen en su atención adaptándose a las características sociolingüisticas del entorno.

\section{Objetivos}

El objetivo general del trabajo es la evaluación del impacto de un proyecto ApS en la formación de competencias generales y específicas de 20 estudiantes del Grado de Logopedia que participan en la elaboración, aplicación y evaluación de un proyecto de ApS.

Como objetivos específicos, se pretende:

-Evaluar y comparar competencias específicas adquiridas por los alumnos que participan en un proyecto ApS respecto a alumnado no participante (el estudiante es capaz de evaluar de una forma adecuada los pares craneales implicados en el habla).

- Evaluar y comparar las competencias generales adquiridas por los alumnos que participan en un proyecto $\mathrm{ApS}$ respecto a alumnado no participante (el estudiante trabaja con el paciente de una manera educada, cuidando de manera específica las formas y adaptándose al nivel y características del paciente, muestra respeto en el trato, sabe escuchar e interpretar el lenguaje no verbal, comunica correctamente y comunica adecuadamente las conclusiones de la exploración).

\section{Desarrollo de la innovación}

El presente proyecto se centra en una propuesta de intervención basada en la metodología educativa del ApS para la asignatura Neurología Clínica aplicada a la Logopedia impartida en el $2^{\circ}$ curso del Grado de Logopedia. El proyecto se encuentra dentro del programa B, Renovación de metodologías docentes en el marco de las ayudas para el desarrollo de proyectos de innovación educativa y mejora de la calidad docente de la Universitat de Valencia. Dentro del programa B, el proyecto se encuentra en la categoría RMD-ESTIC, donde el equipo de trabajo está formado por un profesor docente investigador de la UV (PDI) y cuatro estudiantes del Grado de Logopedia y un estudiante de Logopedia recién egresado. El objetivo de este tipo de ayudas se centra en el trabajo cooperativo del proceso enseñanzaaprendizaje, con acciones de soporte y orientación del estudiante en su proceso de aprendizaje. 
El proyecto se implementó en sustitución a la sesión de 3 horas de prácticas que se desarrollan de forma ordinaria en las instalaciones de la Facultad. Debido al gran volumen de alumnado (unos 40 alumnos por sesión práctica), el proyecto fue una opción voluntaria para los alumnos que deseaban participar. El proyecto se desarrolló para 20 estudiantes. Debido a que el número de solicitudes fue superior al número de plazas ofertadas (54 solicitudes), se procedió al sorteo de las solicitudes. Los alumnos que no deseaban participar o no salieron en el sorteo desarrollaron la práctica de forma habitual, aplicando la propedéutica clínica básica a sus compañeros de clase.

Las actividades vinculadas a los objetivos del proyecto ApS son:

Los alumnos (por parejas) prepararon y practicaron con sus compañeros -antes de la intervención- los diferentes apartados de la anamnesis y la exploración neurológica, prestando atención a las posibles dificultades y las características de las personas mayores.

Los 20 alumnos voluntarios se desplazaron, junto con los alumnos colaboradores y el profesor responsable, a la sede de la Cruz Roja donde pusieron en práctica la propedéutica clínica básica con las fichas diseñadas por el profesor y los alumnos colaboradores (OrtizMasià, 2018; Pérez Gil, 2018). El desplazamiento fue en transporte público.

El proyecto ApS consistía en realizar una exploración neurológica a personas de la tercera edad en situación de desamparo. El encuentro tuvo lugar en la sede central de la Cruz Roja de Valencia. Durante la implantación de proyecto ApS en el curso 2018-2019 se realizó una evaluación de las competencias específicas y generales. Con tal fin se clasificaron los estudiantes en dos grupos:

a) grupo de estudiantes que participaron en el proyecto ApS (ApS) (n=20);

b) grupo de estudiantes que no participaron en el proyecto ApS (NOApS) (n=20).

Una vez realizados los grupos, se compararon las competencias generales y específicas adquiridas en el curso teórico y tras la implantación del proyecto ApS. La evaluación de la adquisición de las competencias generales (Tabla 1 de la 6 a la 12) y específicas (Tabla 1 de la 1 a la 5) se realizó mediante observación directa de la exploración de los pares craneales (PC) en aula y/o en la Cruz Roja y posterior cumplimentación de una rúbrica que contenía competencias específicas y generales (ver Tabla 1 ).

\section{Análisis estadístico de los datos}

Los datos fueron expresados como media \pm Error Estándar de la Media (EEM) del grupo (n $\geq 15$ en todos los grupos) y fueron comparados mediante la prueba t de Student desapareada (Graph-Pad Sotware 6.0). Si el P-valor es $<0,05$ se consideran las diferencias entre hipótesis nula y alternativa como significativas.

\section{Aprobación ética}

Se obtuvo el consentimiento informado de todos los alumnos. 
Tabla 1. Rúbrica de evaluación competencias generales y específicas utilizadas para comparar la adquisición de competencias en los grupos ApS y control.

\begin{tabular}{|c|c|c|c|c|c|c|c|}
\hline & & 0 & 1 & 2 & 3 & 4 & 5 \\
\hline 1. & Explora de manera eficiente el trigémino (V) & & & & & & \\
\hline 2. & Explora de manera eficiente el nervio facial (VII) & & & & & & \\
\hline 3. & Explora de manera eficiente el nervio glosofaríngeo (IX) & & & & & & \\
\hline 4. & Explora de manera eficiente el nervio vago $(X)$ & & & & & & \\
\hline 5. & Explora de manera eficiente el nervio hipogloso (XII) & & & & & & \\
\hline & $\begin{array}{l}\text { Trabaja de manera educada, cuidando de manera específica las } \\
\text { formas y adaptándose al nivel y características del paciente }\end{array}$ & & & & & & \\
\hline 7. & Muestra siempre un respeto exquisito al trato con el paciente & & & & & & \\
\hline 8. & Sabe escuchar sin interrumpir & & & & & & \\
\hline 9. & Sabe interpretar el lenguaje no verbal del paciente & & & & & & \\
\hline 10. & Muestra autocontrol emocional en las interacciones & & & & & & \\
\hline & $\begin{array}{l}\text { Comunica correctamente y fundamentada profesionalmente, } \\
\text { de manera oral sus observaciones y conclusiones }\end{array}$ & & & & & & \\
\hline & $\begin{array}{l}\text { Evita comentar información reservada que no sea } \\
\text { estrictamente necesaria para el caso }\end{array}$ & & & & & & \\
\hline
\end{tabular}

0- No ha realizado el ejercicio.

1- Ha realizado el ejercicio pero con errores sustanciales.

2- Ha realizado el ejercicio con algún error.

3- Ha realizado el ejercicio correctamente.

4- Ha realizado el ejercicio notablemente.

5- Ha realizado el ejercicio de una forma excelente.

\section{Resultados}

\section{Los alumnos que participan en el proyecto ApS mejoran en las competencias especificas reforzadas durante la implantación del proyecto}

Con el fin de conocer la adquisición de competencias específicas relacionadas con la exploración de los pares craneales y reforzadas durante el proyecto ApS se compararon los resultados de la evaluación entre alumnos que habían participado (ApS) y alumnos control (no participantes). Los resultados mostraron que los alumnos ApS presentaban una mejoría significativa en la forma de explorar los parea craneales VII, IX, X y XII. (Figura 1). Entre todos los pares craneales que mostraron una mayor mejoría se encontraban el par craneal IX y X que se evalúan conjuntamente y que ambos están relacionados con la motilidad faríngea y laríngea. Sin embargo, no mostraron mejoría en la exploración del par craneal V, relacionado con la sensibilidad de la cara. Este par craneal requiere de una mayor colaboración del paciente y tiene un componente subjetivo. 


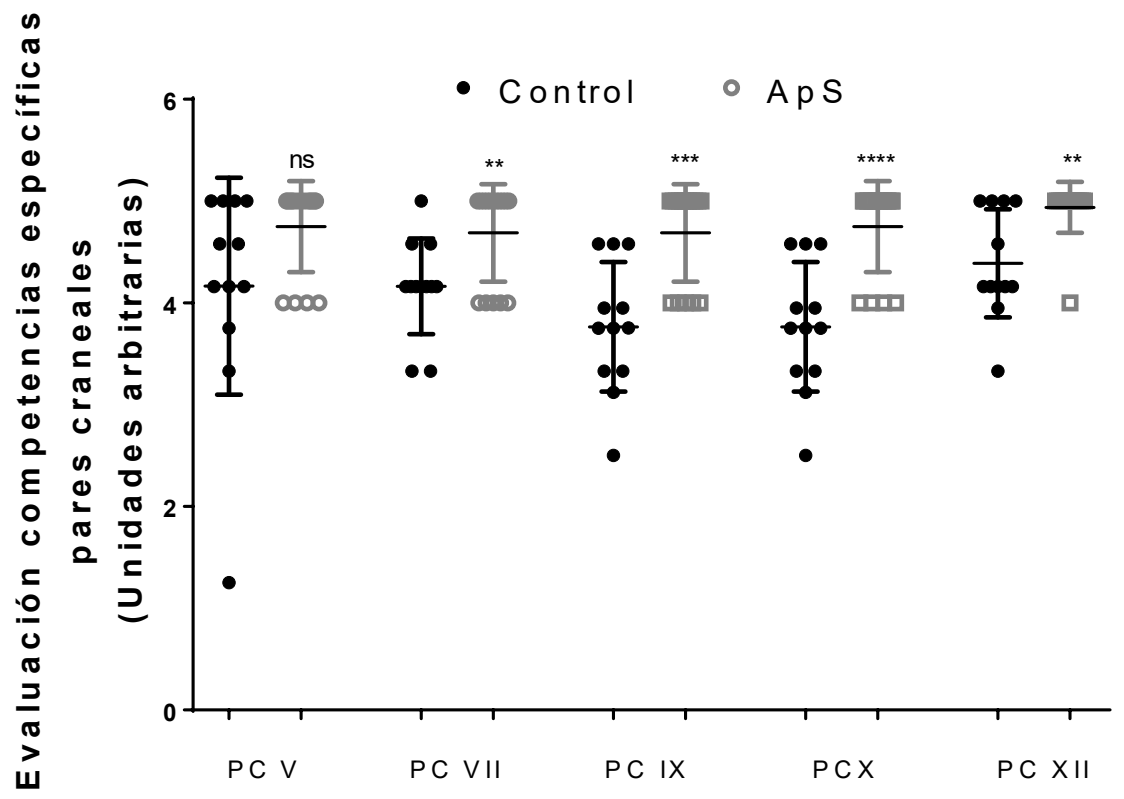

Fig 1. Comparación de las calificaciones obtenidas en alumnos que participaron o no en el proyecto ApS. Evaluación de los PC V, VII, IX, Xy XII. Las barras representan la media \pm EEM del grupo ( $n \geq 15$ en todos los grupos). Las diferencias significativas vs grupo control se muestran como $*^{*} p<0,005$ vs control; $* * * p<0,001$ vs control; $* * * * p<0,0001$ vs control.

\section{Los alumnos que participan en el proyecto ApS mejoran en competencias generales.}

Con el fin de conocer si el proyecto ApS potenciaba la adquisición de competencias generales se compararon los resultados de la evaluación de algunas de las competencias generales entre alumnos que habían participado (ApS) y alumnos control (no participantes). Los resultados mostraron que los alumnos ApS presentaban una mejoría significativa en competencias tales como trabajar de manera educada, el cuidando de manera específica las formas y adaptándose al nivel y características del paciente; mostrar respeto en el trato; saber escuchar e interpretar el lenguaje no verbal; comunicar correctamente y la comunicación correcta de las conclusiones de la exploración (Figura 2). Sin embargo, los alumnos no mejoraron respecto al grupo control en el autocontrol emocional en la interacción y en la competencia relacionada con el secreto profesional. En ambas competencias generales tanto grupo control como ApS se encontraban en puntuaciones máximas. 


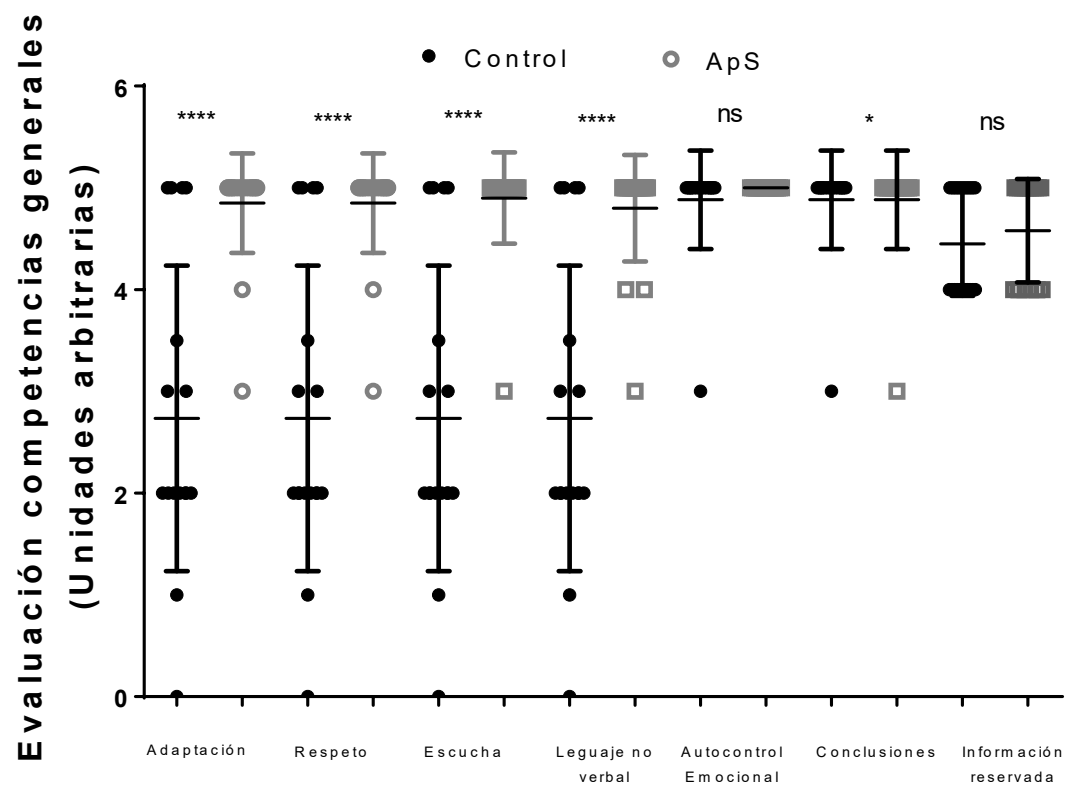

Fig 2. Comparación de competencias generales entre alumnos que participaron o no en el proyecto ApS. Las barras representan la media \pm EEM del grupo ( $n \geq 15$ en todos los grupos). Las diferencias significativas $v s$ grupo control se muestran como $* p<0,05$ vs control; $* * * *<<0,0001$ vs control.

\section{Conclusiones}

La implantación del proyecto de ApS en los alumnos de segundo del Grado de Logopedia reforzó de forma adecuada las competencias específicas relacionadas con la exploración de los PC relacionados con el habla, especialmente los PC IX y X, que son los que presentan mayor dificultad de exploración. Sin embargo, en la exploración del PC V el grupo ApS no mostró mejoría con respecto al grupo control. Una posible explicación es que el quinto PC presenta un componente subjetivo muy importante que los ancianos no supieron transmitir y/o los alumnos evaluar correctamente. En relación a las competencias generales, el proyecto ApS fomentó de forma adecuada competencias tan importantes como trabajar de manera educada, el cuidando de manera específica las formas y adaptándose al nivel y características del paciente; mostrar respeto en el trato; saber escuchar e interpretar el lenguaje no verbal; comunicar correctamente y la comunicación correcta de las conclusiones de la exploración. Todas ellas son competencias que se adquieren con la experiencia y específicamente fomentando la empatía con el paciente que en un aula es difícil de conseguir pero que el proyecto ApS ayudó en la adquisición.

\section{Agradecimientos}

Innovation Project Universitat de Valencia nº UV-SFPIE_RMD18-841128.

\section{Referencias}

CASADO DE LA GALA, L. (2015). Aprendizaje servicio, proceso de mejora para la ciudadanía. Palencia: Facultad de Educación de Palencia, Universidad de Valladolid. 
MARTÍNEZ, M. ET AL. (2010). Aprendizaje servicio y responsabilidad social de las Universidades. Barcelona: Ediciones Octaedro, S.L. y Universitat de Barcelona.

RODRÍGUEZ GALLEGO, MR (2014). El Aprendizaje-Servicio como estrategia metodológica en la Universidad. Revista Complutense de Educación. Vol. 25 Núm. $195-$ 113

\section{Ponencias de congresos}

ORTIZ-MASIÀ, D (2018). “Aplicación de la metodología ApS en alumnos del Grado de Logopedia. Jornadas” IDES 2018.

PÉREZ GIL, T, LAHIGUERA LUJAN, C, BAUTISTA PÉREZ, MJ, TRIGUERO TARAZONA, S BAGUENA MANCEBO, S Y ORTIZ-MASIÀ, D (2018). "Aplicación de la metodología ApS en alumnos del Grado de Logopedia: intervención con personas de la tercera edad". Congreso In-Red 2018. Libro de Actas pagina 461-470

\section{Página web}

Portal oficial European Higher Education Area and Bologna process. https://www.ehea.info/ [Consulta: 17 de mayo de 2019]

Portal official Grado Logopedia de la Universitat de València: https://www.uv.es/uvweb/grado-logopedia/es/grado-logopedia-1285929409765.html

[Consulta: 17 de mayo de 2019]

\section{Legislación y normas}

España. Orden CIN/726/2009, de 18 de marzo, por la que se establecen los requisitos para la verificación de los títulos universitarios oficiales que habiliten para el ejercicio de la profesión de Logopeda. BOE. 26 de marzo de 2009, núm. 73, p. 29159-29163. 